\title{
Stable Registration of Pathological 3D SD-OCT Scans using Retinal Vessels
}

\author{
Jing $\mathrm{Wu}^{1}$ *, Bianca S. Gerendas ${ }^{1}$, Sebastian M. Waldstein ${ }^{1}$, Georg Langs ${ }^{2}$, \\ Christian Simader ${ }^{1}$, and Ursula Schmidt-Erfurth ${ }^{1}$ \\ ${ }^{1}$ Christian Doppler Laboratory for Ophthalmic Image Analysis, Department of \\ Ophthalmology and Optometry, Medical University of Vienna, Austria \\ 2 Computational Imaging Research Lab, Department of Biomedical Imaging and \\ Image-guided Therapy, Medical University of Vienna, Austria
}

\begin{abstract}
We propose a multiple scanner vendor registration method for pathological retinal 3D spectral domain optical coherence tomography volumes based on Myronenko's Coherent Point Drift and our automated vessel shadow segmentation. Coherent point drift is applied to the segmented retinal vessel point sets used as landmarks to generate the registration parameters required. In contrast to other registration methods, our solution incorporates a landmark detection and extraction method that specifically limits the extraction of false positives and a registration method capable of handling any such noise in the landmark point sets. Our experiments show modified Hausdorff distance is reduced by a minimum of $91 \%$ between target and registered vessel point sets with at least $94 \%$ of bifurcations correctly overlapping based on ground truth, a significant improvement over current methods.
\end{abstract}

\section{Introduction}

Spectral-domain Optical Coherence Tomography (SD-OCT) is a non-invasive modality for acquiring high resolution, 3D cross sectional volumetric images of the retina and the sub-retinal layers, including retinal pathology. Today SD-OCT is the most important ancillary test for the diagnosis of sight degrading diseases such as age-related macular degeneration (AMD) and glaucoma [1]. Disease diagnosis, assessment, and treatment requires a patient to undergo multiple OCT scans, possibly using different scanning devices to gauge disease activity, progression and treatment success. However, this scanning process, combined with patient motion may result in poor scan spatial correlation, potentially leading to incorrect patient diagnosis or treatment analysis. Image registration has been applied to solve this problem using iterative closest point (ICP) in [2] to compare disease states by registering different volumes. However, a major limitation of ICP is the assumption that every source point corresponds with the closest

* The Financial support of the Austrian Federal Ministry of Economy, Family and Youth and the National Foundation for Research, Technology and Development is gratefully acknowledged. 
point to it in the target point set which, in retinal OCT, cannot be assumed in all cases. Accurate and reproducible landmarks are required to register 3D scans from different time-points and vendors, the most suitable being the retinal vasculature. The authors in [2] apply a landmark detection and point based registration method that is untested on wet-AMD (used here, a leading cause of blindness due to blood vessel leakage, opposed to the more common dry-AMD) and is validated on only a single vendor. In addition, as far as we are aware, other works in this area focus on OCT to fundus registration $[3,4]$ where also healthy cases were used.

In Sec. 2 we present an automated retinal vessel segmentation method for landmark acquisition, less susceptible to pathology, and in Sec.3 register using coherent point drift (CPD), suitable for noisy point sets with limited overlap and similarity. Our approach is evaluated in Sec.4 on scans from 3 major OCT vendors (Zeiss Cirrus, Nidek RS3000 \& Topcon 3D 2000). We show that in addition to being suitable for multiple OCT vendors and reproducible (Fig.3), our method is accurate based on expert analysis of resulting registered retinal vessels and shows improved performance over current methods.

\section{Automated Landmark Extraction}

We define an OCT volume as $\mathbf{V}(Z, X, Y)$ were $Z$ is the axial, $X$ the primary and $Y$ the secondary scan directions and is comprised of B-scans slices $\mathbf{B}_{s}(Z, X)$ (2D images perpendicular to fundus where $X$ is the horizontal axis and $Z$ is the vertical axis). Volume dimensions range up to $1024 \times 512 \times 256$ voxels, covering $6 \mathrm{~mm}^{3}$. We denote the retinal pigment epithelium layer (RPE, the bright layer in the lower retina) for a given B-scan as having a lowest outer surface point $R P E S_{\min }$ and the difference $\left(R P E S_{d i f f}\right)$ between $R P E S_{\min }$ and the original $\mathrm{RPE}$ outer surface position $R P E S_{\text {orig }}$ for a given $X$ column point.

\subsection{OCT Scan Pre-processing}

Retinal vessels are not clearly visible within SD-OCT scans due to high attenuation of blood. However, shadows are generated as a result of the absorption of light transmitted from the scanner, visible most prominently in the RPE layer, and used here to locate the retinal vessels [5]. Firstly each $\mathbf{B}_{s}$ is "flattened" to accurately and reproducibly locate the RPE layer and secondly a composite "projection" image $\mathcal{P}$ is computed from the RPE layers. Flattening adjusts a given $\mathbf{B}_{s}$ in the $Z$ axis such that the outer RPE surface has the same $Z$ value across the $X$ axis, compensating for varying retinal curvatures across different $\mathbf{B}_{s}$. Using the vendor RPE surface segmentation, each column in $\mathbf{B}_{s}$ is shifted in $Z$ by $R P E S_{\text {diff }}$, flattening the RPE surface as in Fig.1(a).

A cross-section $\left(R P E_{C S}\right)$ is taken from the flattened $\mathbf{B}_{s}$ covering $20 \mu \mathrm{m}$ from the outer RPE surface towards the inner limiting membrane (ILM, the upper most surface of the retina), based on examination of RPE thickness from 
21 training scans across 3 vendors ensuring the clinically significant RPE is included. Averaging the column intensities within $R P E_{C S}$ improves vessel shadow visibility [5] resulting in a single line profile $\left(R P E_{L P}\right)$ representing the RPE layer for a given $\mathbf{B}_{s}$. This process is repeated for each $\mathbf{B}_{s}$ creating a composite image $\mathcal{P}(X, Y)$ (where $Y$ is the vertical axis) from all $R P E_{L P}$ ranging in dimensions from $512 \times 128$ to $256 \times 256$ pixels.

\subsection{Projection Denoising}

Each $R P E_{L P}$ discussed in Sec.2.1 features a degree of speckle noise [6] which hinders the detection system. A block matching based sparse transform domain collaborative filtering approach [7], applied to $\mathcal{P}$, has shown to be effective. An initial basic estimate is calculated by first taking block-wise estimates where, for each block within the image, grouping of similar blocks to the current block generates a 3D array. Collaborative hard-thresholding is applied to the 3D transformed array coefficients to attenuate the noise. Estimates of the grouped blocks are obtained by inverting the 3D transform which are then returned to their original locations within the image. The basic estimate is computed by weighted averaging $\left(w_{x_{R}}^{h t}\right)$ of all the block-wise estimates that overlap. A final estimate is then calculated from the basic estimate. For each block, two 3D arrays are created using block-matching, one from the noisy image and one from the basic estimate. A 3D transform is applied on both arrays in addition to a Wiener filter applied to the noisy array using the energy spectrum of the basic estimate. Estimates of all grouped blocks are generated from the inverse 3D transform on the filtered coefficients before returning the block estimates to their original positions within the image. Finally a weighted average $\left(w_{x_{R}}^{w i e}\right)$ is used to aggregate all the local estimates to compute the final estimate $\left(\hat{y}^{\text {final }}\right)$ as described by Eqn.1 where $\chi_{x_{m}}: X^{\prime} \rightarrow 0,1$ is the characteristic function of the square support of a block located at $x_{m} \in X^{\prime}$ and $\hat{Y}^{\prime}{ }_{x_{m}}^{\text {wie, } x_{R}}$ are the block-wise estimates.

$$
\hat{y}^{\text {final }}(x)=\frac{\sum_{x_{R} \in X^{\prime}} \sum_{x_{m} \in S_{x_{R}}^{w i e}} w_{x_{R}}^{\text {wie } \hat{Y}^{\prime}{ }_{x_{m}}^{\text {wie } x_{R}}(x)}}{\sum_{x_{R} \in X^{\prime}} \sum_{x_{m} \in S_{x_{R}}^{\text {wie }}} w_{x_{R}}^{\text {wie }} \chi_{x_{m}}(x)}, \forall x \in X^{\prime},
$$

An $\alpha$ parameter is specified depending on the level of noise to define the block size used. Noise filtering with $\alpha=20$ (specifying a relatively small block size) was found to be most suitable for removing speckle noise while maintaining the integrity of vessel shadows in cross vendor experiments as seen in Fig.1(c) compared to the original $\mathcal{P}$ in Fig.1(b).

\subsection{Pathology Removal \& Tiling}

In addition to noise, the presence of non-vessel shadows caused by pathology adversely affects the detection system and their removal is required to prevent their usage as landmarks. Such shadows appear in $\mathcal{P}$ with similar intensity to 


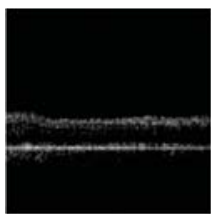

(a)

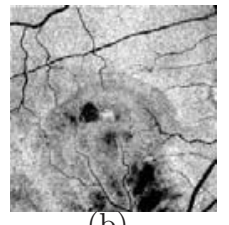

(b)

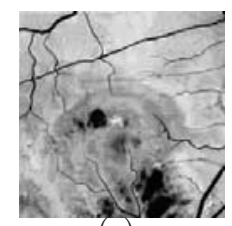

(c)

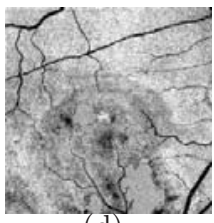

(d)

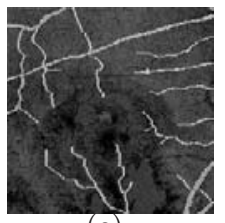

(e)

Fig. 1. (a) Exemplar flattened $\mathbf{B}_{s}$. (b) Exemplar original $\mathcal{P}$ with (c) denoised $\mathcal{P}$ using $\alpha=20$. (d) $\mathcal{P}$ from (b) \& (c) with pathology shadow removed. (e) Segmented vessel shadows overlayed onto $(d)$.

vessel shadows but generally possess different size and shape characteristics. Vessels are generally long, thin connected regions, thus the opposite characteristics can be used to describe pathology shadows. However, due to imaging artefacts at acquisition and patient motion, the shadows can become distorted and may feature similar characteristics. Thus to distinguish between these two sets of shadows, the aforementioned shape characteristics were used as a feature, given that pathology shadows are amorphous and not thin and narrow. In addition, they seldom reach the boundaries of $\mathcal{P}$ and are are not simultaneously long, thin and with a low area. For each detected pathology shadow region, randomly selected intensities from the object boundary are used to fill the pathology region, masking it from $\mathcal{P}$ (Fig.1(d)) while maintaining a similar appearance to the background. This is repeated for all pathology regions at which point the masked $\mathcal{P}$ is denoised again giving $\mathcal{P}_{M}$.

Due to the presence of pathology, motion artefacts and noise, intra-projection intensity and appearance variation is present. Such issues can be limited by reducing the individual vessel detection window using tiling, denoted as $\mathcal{P}_{M T}$. Thus the search space is reduced to equally sized, non-overlapping tiles in $4 \times 4$, $2 \times 2,2 \times 1$ and $1 \times 2$ patterns covering the entire $\mathcal{P}$. Multiple grids are used to ensure that the vessels are intact within at least one of the grid sizes.

\subsection{Vessel Shadow Detection}

The vessel enhancement filter proposed in [8] is applied on the $\mathcal{P}_{M T}$ sub-images to detect tubular geometric structures and suppress remaining noise and background. Applied to $\mathcal{P}_{M T}$, the Taylor expansion approximates the image structure to the second order resulting in the gradient vector $\left(\nabla_{o, s}\right)$ and Hessian matrix $\left(\mathcal{H}_{o, s}\right)$ computed in $x_{o}$ at scale $s$. Using eigenvalue analysis of the Hessian, the principal directions in which the local second order image structure is decomposed are extracted. This results in three orthonormal directions giving a spherical neighbourhood centred at $x_{o}, \mathcal{N}_{x_{o}}$, mapped by $\mathcal{H}_{o}$ to an ellipsoid with directions given by the Hessian eigenvectors and axis semi-lengths as the magnitudes of eigenvalues. This ellipsoid describes the second order structure where eigenvalues are ordered $\left|\lambda_{1}\right| \leq\left|\lambda_{2}\right| \leq\left|\lambda_{3}\right|$. Two geometric ratios based on the second order ellipsoid are used as a dissimilarity measure. The first defines the deviation from blob-like structures whereas the second distinguishes between 


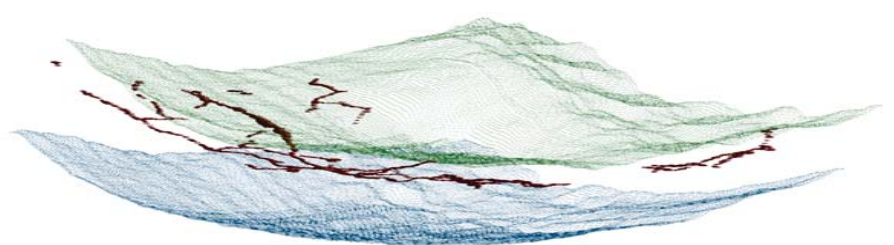

Fig. 2. Exemplar retinal vessel segmentation showing modelled in red the vessels between the green ILM surface points and blue RPE surface points.

plate-like and line-like structures. Thus a measure defining second order structuredness of an image is used to distinguish between background and vessel. The authors explain this measure will be low for the background and larger in regions of vessels, however the opposite would be true for OCT projection images. This is due to the vessel shadows appearing dark against a lighter background whereas the method in [8] was originally proposed for MRA and CTA images which are commonly interpreted using maximum intensity projection and feature brighter vessels against darker background. However the dark-to-light distinction is still present and prominent in $\mathcal{P}_{M T}$, as well as similarities such as vessel structures occupying a small volume of the image as a whole.

Region growing using the highest intensity pixel from the result of the vessel enhancement filter segments the vessel shadow(s) within each tile of $\mathcal{P}_{M T}$. This process is repeated for each combination of tile patterns, with the intersection of all candidate segmentations taken as the final result. This can be seen in Fig.1(e) where the segmented vessel shadows are overlayed onto $\mathcal{P}_{M}$.

\subsection{Segmented Vessel Point Post Processing}

The vessel shadows obtained from $\mathcal{P}$ are a $2 \mathrm{D}$ representation, however the vessel structure is $3 \mathrm{D}$ and the third dimensional spatial information is important for volume registration. The segmented vessel shadows are skeletonized by removal of the boundary pixels until only a single pixel remains along the structure while preventing the structure from breaking apart. The $X$ and $Y$ coordinate values for each vessel shadow skeleton point are compared with the corresponding coordinates from the original vendor segmented ILM to identify the relative depth $(Z)$ position within the OCT volume. This can be visualized as shown in Fig.2.

\section{Point Based Vessel Tree Registration}

In this section, we show the use of CPD registration to align retinal OCT volumes using the landmarks identified in Sec.2.

\subsection{Point Set Registration with CPD}

The alignment of two point sets, defined here as the source and target 3D retinal vessel centrelines obtained in Sec.2, is considered in CPD as a probability den- 
sity estimation problem by [9]. One point set represents the Gaussian mixture model (GMM) centroids and the other represents the data points. The two point sets become aligned at the optimum and the correspondence is defined by the maximum of the GMM posterior probability for a given data point.

Given the GMM centroid points and the data points generated by the GMM, the GMM probability density function (PDF) is defined. In addition, the authors in [9] also add a weighted uniform distribution to the mixture model allowing it to account for noise and outliers. For all GMM components, equal isotropic covariances $\sigma^{2}$ is used as well as equal membership properties. The GMM centroid locations are re-parametrized using the parameter set $\theta$, estimated using maximum likelihood allowing the i.i.d data assumption to be made. Thus the correspondence probability between two points can be defined as the posterior probability of the GMM centroid. Expectation Maximization (EM) is used to find $\theta$ and $\sigma^{2}$ where as the expectation (E) step, the parameters $\theta$ and $\sigma^{2}$ are initially guessed and then the Bayes' theorem is used to compute a posteriori probability distributions of mixture components. The maximisation (M) step is then used to find the new parameter values by minimizing the expectation of the complete negative log-likelihood function with respect to the new parameters. The $\mathrm{E}$ and $\mathrm{M}$ steps are processed alternately until convergence, generating the posterior probabilities of the GMM components. Unlike ICP, in CPD, the GMM centroids are forced to move coherently as a group to preserve the topological structure of the point sets.

We examine the affine $\left(\tau_{A}\right)$ and non-rigid $\left(\tau_{N}\right)$ registrations here. For $\tau_{A}$, optimization is unconstrained and defined as $\mathcal{T}\left(y_{m} ; \mathbf{R}, \mathbf{t}, s\right)=\mathbf{B y}_{m}+\mathbf{t}$, where $\mathbf{B}_{D \times D}$ is an affine transformation matrix and $\mathbf{t}_{D \times 1}$ is the translation vector. For $\tau_{N}$, transformation is defined as the initial position plus a displacement function, $\mathcal{T}(\mathbf{Y}, v)=\mathbf{Y}+v(\mathbf{Y})$, where $v$ is the displacement function. In the method for $\tau_{N}$, the authors in [9] regularize the norm of the displacement function. This approach is based on the motion coherence theory (MCT) [10] stating that points close to one another have a tendency to move coherently, thus the displacement function between points sets should be smooth. The regularization term in [9] is equivalent to the MCT, implying that point based motion coherence is imposed. This regularization is integral to CPD and its ability to register point sets coherently, important for retinal vessel registration. Thus we use a high regularization parameter for $\tau_{N}$ to maintain vessel structure coherence. A transformation matrix is then generated from the registered vessel points. Currently only affine transformation is used as the clinical suitability of further transformations affecting the structure of the retina by deformation is uncertain.

\section{Registration Results}

To evaluate registration performance, two sets of results are presented. In our experiments, intra-vendor registration was carried out between pairs of OCT scans acquired from 8 patients at different time points (inter-vendor registration differs only in that the landmarks come from scans of distinct vendors). 7 pairs 

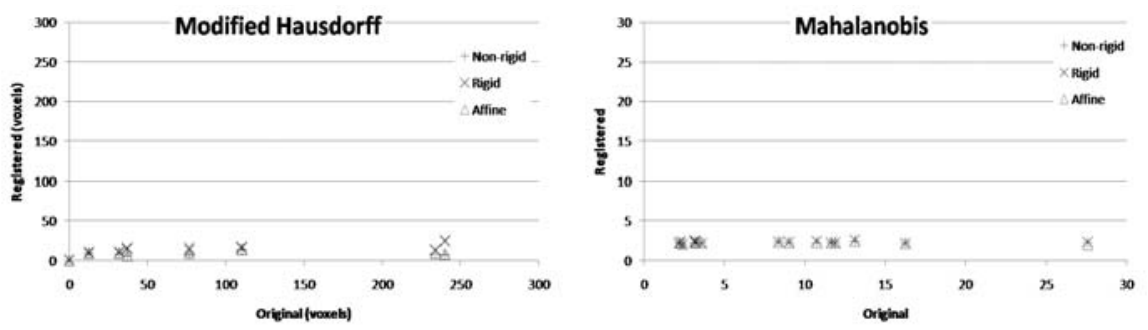

Fig. 3. (a) MHD \& (b) MD between pairs of registered OCT scans comparing distances prior to registration (horizontal axis) against distances post registration (vertical axis).

feature unique scans and the eighth pair was used as a control and featured one unique scan twice. Thus a total of 15 unique 3D SD-OCT fovea-centred diseased volumes were used for testing. Of the 15 scans, levels of pathology ranged from low to high primarily due to the presence of cysts, exudates and drusen. In addition, motion artefacts in the $X$ direction are seen in $\mathcal{P}$ due to patient motion, resulting in misaligned $\mathbf{B}_{s}$.

Firstly, we used CPD to register between scan pairs in $3 \mathrm{D}$ using $\tau_{N}$ and $\tau_{A}$. Registration performance was quantified by calculating the modified Hausdorff [11] (MHD, Fig.3) and Mahalanobis distances (MD, Fig.3) between point sets prior and post registration, where point set distance is expected to decrease post registration as the retinal vessels become aligned. Prior to registration, a mean MHD of $92.89 \pm 92.41$ voxels was calculated for the vessel pairs. This was reduced to $8.319 \pm 3.91$ and $7.579 \pm 3.569$ voxels for $\tau_{A}$ and $\tau_{N}$ respectively after registration where. A mean MD of $8.192 \pm 6.999$ prior to registration for the vessel pairs was reduced to $2.259 \pm 0.078$ and $2.249 \pm 0.109$ for $\tau_{N}$ and $\tau_{A}$ respectively after registration. Thus registration reduced the mean MHD across all scan pairs by $92 \% \& 91 \%$ for $\tau_{N}$ and $\tau_{A}$ respectively and mean MD was reduced by $72 \% \& 73 \%$ for $\tau_{N}$ and $\tau_{A}$ respectively. Comparison with the state of the art method proposed in [2] applied to the same test scans resulted in mean MHD of $94.39 \pm 93.33$ voxels and mean MD of $7.804 \pm 5.373$ between registered and target scans, showing our method to perform significantly better.

Our second experiment qualitatively assesses system performance to account for the disjoint segments of the landmark retinal vessels between scan pairs. The registration results were given to 3 expert graders who were asked to identify well and poorly overlapping major bifurcations between the target and registered vessel points. Across the graders, a mean of $136 \pm 7$ and $4 \pm 1$ bifurcations using $\tau_{A}$ were well and poorly aligned respectively. Using $\tau_{N}$, this became a mean of $139 \pm 10$ and $2 \pm 1$ bifurcations. Thus $94 \%$ and $96 \%$ of bifurcations correctly overlapped between target and registered vessel sets using $\tau_{A}$ and $\tau_{N}$ respectively.

\section{Conclusion}

We have proposed a method of retinal OCT volume registration using CPD registration of segmented retinal vessel shadows as landmarks. We first showed that 
the retinal vessels could be used to accurately and reproducibly represent the retinal OCT volume for both intra-patient acquisition and multi-vendor acquisition. By combining these segmented vessel shadows and modelling them as the retinal vessels, based on their geometric parameters obtained from the respective volume and layer information, CPD registration was applied to transform the temporally acquired vessel point sets. Our experiments quantifying the MHD and MD between target and registered point sets show the minimisation of the distances between original and target point sets after transformation, by up to $92 \%$ using non-rigid transformation and 91\% using affine transformation. Qualitative analysis of the transformed point set bifurcations with their respective targets by experienced graders shows a mean of $96 \%$ correctly overlapping bifurcations when using non-rigid transformation. In addition, comparison against current state of the art methods for retinal OCT volume registration show significantly improved performance for pathological, multiple vendor scans.

\section{References}

1. Geitzenauer, W., Hitzenberger, C.K., Schmidt-Erfurth, U.M.: Retinal optical coherence tomography: past, present and future perspectives. Br J Ophthalmol 95 (July 2010) 171-7

2. Niemeijer, M., Lee, K., Garvin, M.K., Abràmoff, M.D., Sonka, M.: Registration of $3 \mathrm{~d}$ spectral oct volumes combining ICP with a graph based approach. In: SPIE Medical Imaging. Volume 8314. (February 2012)

3. Kolar, R., Tasevsky, P.: Registration of 3D Retinal Optical Coherence Tomography Data and 2D Fundus Images. BIR 6204 (2010) 72-82

4. Golabbakhsh, M., Rabbani, H.: Vessel-based registration of fundus and optical coherence tomography projection images of retina using a quadratic registration model. IET Image Processing 7 (2013) 768-76

5. Niemeijer, M., Garvin, M.K., van Ginneken, B., Abràmoff, M.D.: Vessel segmentation in $3 \mathrm{~d}$ spectral oct scans of the retina. In: SPIE Medical Imaging. Volume 6914. (March 2008)

6. Schmitt, J.M., Xiang, S.H., Yung, K.M.: Speckle in optical coherence tomography. J. Biomed. Opt. 4 (1999) 95-105

7. Dabov, K., Foi, A., Katkovnik, V., Egiazarian, K.: Image Denoising by Sparse 3D Transform-Domain Collaborative Filtering. IEEE Trans on Image Proc 16(8) (August 2007)

8. Frangi, A., Niessen, W.J., Vincken, K.L., Viergever, M.A.: Multiscale vessel enhancement filtering. MICCAI 1496 (1998) 130-37

9. Myronenko, A., Song, X.: Point Set Registration: Coherent Point Drift. PAMI 32 (2010) 2262-2275

10. Yuille, A.L., Grzymacz, N.M.: The motion coherence theory. ICCV 3 (1988) 344-353

11. Dubuisson, M., Jain, A.K.: A Modified Hausdorff Distance for Object Matching. ICPR 1 (1994) 566-568 